\title{
Volume Integral Mean of Holomorphic Function on Polydisc
}

\author{
Lijuan Xu ${ }^{1,}$, , Hua Liu ${ }^{1}$, Juan Chen ${ }^{2}$, Xiaoli Bian ${ }^{1}$ \\ ${ }^{1}$ College of Science, Tianjin University of Technology and Education, Tianjin, China \\ ${ }^{2}$ Basic Courses Department, Tianjin Sino-German University of applied Science, Tianjin, China
}

\section{Email address:}

hbhdxlj@163.com (Lijuan Xu), hualiu@tute.edu.cn (Hua Liu), daliuhua@163.com (Hua Liu), chenjuan@tsguas.edu.cn (Juan Chen), bianxiaoli@yeah.net (Xiaoli Bia)

*Corresponding author

\section{To cite this article:}

Lijuan Xu, Hua Liu, Juan Chen, Xiaoli Bian. Volume Integral Mean of Holomorphic Function on Polydisc. International Journal of Theoretical and Applied Mathematics. Vol. 6, No. 1, 2020, pp. 14-18. doi: 10.11648/j.ijtam.20200601.12

Received: December 17, 2019; Accepted: January 4, 2020; Published: January 13, 2020

\begin{abstract}
Let $f$ be an analytic function in the Hardy space on the polydisc $P_{2}$. In this article we discuss the area integral means $M_{p}(f, r)$ of $f$ on the polydisc $P_{2}$ with radius $r$, and its weighted volume means $M_{p, \alpha}(f, r)$ with to the weight $\left(1-\left|\mathrm{z}_{1}\right|^{2}\right)^{\mathrm{a}} \times\left(1-\left|\mathrm{z}_{2}\right|^{2}\right)^{\mathrm{a}}$. We prove that both $M_{p}(f, r)$ and $M_{p, \alpha}(f, r)$ are strictly increasing in $r$ unless $f$ is a constant. In contrast to the classical case, we also give a example to show that $\log M_{p, \alpha}(f, r)$ is not always convex with respect to $\log r$, although that we still prove that $\log M_{p}(f, r)$ is logarithmically convex.
\end{abstract}

Keywords: Hardy Space, Polydisc, Integral Means, Logarithmically Convex

\section{The Introduction}

Let $f$ be an analytic function on unit circle $\mathrm{D}$ in complex plane $\mathbb{C}$. For $0<p<\infty$ the mean integral of $f$ is defined as

$$
M_{p}(f, r)=\left[\frac{1}{2 \pi} \int_{0}^{2 \pi}\left|f\left(r e^{i \theta}\right)\right|^{p} d \theta\right]^{1 / p}, 0 \leq r<1,
$$

The classical Hardy convex theorem, which is an important tool for complex analysis and harmonic analysis, especially Hardy function space theory, states that $M_{p}(f, r)$ is strictly increasing in $r \in[0,1)$ and $\log M_{p}(f, r)$ is logarithmically convex (see [1]). Xiao and Zhu discussed the extension of these results in the volume integral case in [2]. In fact, they considered the more general unit sphere problem, namely,

$$
M_{p, \alpha}(f, r)=\left[\frac{1}{v_{\alpha}\left(r \mathrm{~B}_{n}\right)} \int_{r \mathrm{~B}_{n}}|f(z)|^{p} d v_{\alpha}(z)\right]^{1 / p}, 0 \leq r<1,
$$

where $\alpha$ is real number and $d v_{\alpha}(z)=\left(1-|z|^{2}\right)^{\alpha} d v(z)$ is a weighted measure on the unit sphere, and concluded
$M_{p, \alpha}(f, r)$ strictly increases in $r$ and however $\log M_{p, \alpha}(f, r)$ of $r$ is logarithmically convex or logarithmically concave depends on the sign of the parameter $\alpha$. Further they proved that $\log M_{p, \alpha}(f, r)$ is logarithmically convex when $\alpha \leq 0$, and a logarithmically concave function when is non-negative.

In this paper, we discuss the case on the polydisc $\mathrm{P}_{2}=\left\{\left|z_{1}\right|<1\right\} \times\left\{\left|z_{2}\right|<1\right\}$. Denote by $\partial_{0} \mathrm{P}_{2}=S_{1} \times S_{2}$ the characteristic boundary. Note that the topology of the characteristic boundary of the polydisc is very different from that of the unit sphere, therefore the function space on $\mathrm{P}_{2}$ has some special properties (see [3]).

Let $f(z)$ be a holomorphic function on $\mathrm{P}_{2}$ and $d \sigma(z)$ be a normalized Borel measures on a unit disk. For $0<p<\infty$, the mean of weighted volume integral of $f(z)$ is defined by

$$
M_{p, \alpha, \beta}(f, r)=\left[\frac{1}{v_{\alpha}\left(r \mathrm{P}_{n}\right)} \int_{r \mathrm{P}_{n}}|f(z)|^{p} d v_{\alpha, \beta}(z)\right]^{1 / p}, 0 \leq r<1
$$

where $d v_{\alpha, \beta}(z)=d \sigma_{\alpha(z)}\left(z_{1}\right) d \sigma_{\beta(z)}\left(z_{2}\right)$ with 


$$
\begin{aligned}
& d \sigma_{\alpha(z)}\left(z_{1}\right)=\left(1-\left|z_{1}\right|^{2}\right)^{\alpha} d \sigma\left(z_{1}\right), \\
& d \sigma_{\beta(z)}\left(z_{2}\right)=\left(1-\left|z_{2}\right|^{2}\right)^{\alpha} d \sigma\left(z_{2}\right),
\end{aligned}
$$

and

$$
v_{\alpha, \beta}\left(r \mathrm{P}_{2}\right)=\int_{r \mathrm{P}_{2}} d v_{\alpha, \beta}(z)
$$

We will only discuss the case when $\alpha=\beta$ in this article. We shall use the notation $v_{\alpha}$ instead of $v_{\alpha, \alpha}$ and $M_{p, \alpha}(f, r)$ instead of $M_{p, \alpha, \beta}(f, r)$. In particular, when $p=\infty$, it can be understood as

$$
M_{\infty}(f, r)=\sup \left\{|f(z)|: z \in \mathrm{P}_{2},|z|=r\right\} L^{p}\left(\mathrm{P}_{2}, d v_{\alpha}\right), 0 \leq r<1
$$

in the usual sense. When $0<p<\infty$ and $\alpha>1$, the weighted Bergman space $\mathrm{A}_{\alpha}^{p}$ is the intersection of holomorphic function spaces $\mathrm{H}\left(\mathrm{P}_{2}\right)$ and $L^{p}\left(\mathrm{P}_{2}, d v_{\alpha}\right)$ on $\mathrm{P}_{2}$, where the norm on $\mathrm{A}_{\alpha}^{p}$ is given by

$$
\|f\|_{p, \alpha}=\left[\int_{\mathrm{P}_{2}}|f(z)|^{p} d v_{\alpha}(z)\right]^{1 / p}
$$

We need some knowledge of the Hardy space. For $0<p<\infty$, the unit polydisc $P_{2}$ is a Hardy space, and $\mathrm{H}^{p}$ is a holomorphic function that contains $\mathrm{P}_{2}$ satisfies the following conditions:

$$
\|f\|_{p}=\sup _{0 \leq r<1} M_{p}(f, r)<\infty,
$$

Where

$$
M_{p}(f, r)=\left[\int_{s_{1} \times s_{2}}|f(r \zeta)|^{p} d \sigma(\zeta)\right]^{1 / p}
$$

is the area integral mean of fand $d \sigma(\zeta)$ is the normalization Lebesgue measure of multi-cylinder surface $\partial \mathrm{P}_{2}$, namely $d \sigma(\zeta)=\frac{1}{(2 \pi)^{2}} d \theta_{1} d \theta_{2}$. It should pointed out here that the

Hardy space on polydisc is quite different from those on spheres. For example, the modulus of functions of Hardy space on n-dimensional complex spheres is integral on real $2 \mathrm{n}-1$ dimensional spheres, while the modulus of function of Hardy space on n-dimensional polydisc is integral on real n-dimensional torus. In this paper, we will discuss the monotonicity of $M_{p}(f, r)$ and $M_{p, \alpha}(f, r)$ and the logarithmical convexity of the corresponding logarithmic functions $\log M_{p}(f, r)$ and $\log M_{p, \alpha}(f, r)$. Since the symmetry of multiple cylinders is not as good as that of unit spheres, the method presented in [2] do not apply to here. But we find a new method to discuss it, and there will be some new conclusions.

\section{Monotonicity of $M_{p}(f, r)$ and $M_{p, \alpha}(f, r)$ and Its Application}

We will firstly consider the case of the Hardy space.

Theorem 2.1 Let $0<p<\infty$ and let $f(z)$ be a holomorphic function with extraordinary values on $\mathrm{P}_{2}$. Then the function $r \mapsto M_{p}(f, r)$ is strictly increasing in the interval $[0,1)$.

Remark. The same theorem on the unit disc has been proved in [4]. This problem on multiple cylinders is not easy to be transformed into the unit disc. It is also di erent from the case on the unit sphere in [2]. The symmetry of multiple cylinders is insu cient to use slice. We need some special handling to prove it.

Proof: Suppose that $f(z)=f\left(z_{1}, z_{2}\right)$ is not a constant function. Then either $f_{z_{1}}\left(z_{2}\right)=f\left(z_{1}, z_{2}\right)$ is non-constant with respect to $z_{2} \in s_{1}$ for some fixed $z_{1}$, or $f_{z_{2}}\left(z_{1}\right)=f\left(z_{1}, z_{2}\right)$ is a non-constant with respect to $z_{1} \in s_{1}$ for some fixed $z_{2}$. Without loss of generality, we might consider the former case. Instead of dealing directly with $M_{p}(f, r)$, let's start with binary real functions of functions $[5]$.

$$
\begin{aligned}
& M_{p}^{p}\left(f, r_{1}, r_{2}\right)=\int_{\partial \mathrm{P}_{2}}\left|f\left(r_{1} \zeta_{1}, r_{2} \zeta_{2}\right)\right|^{p} d s(\zeta) \\
& =\int_{s_{1} \times s_{2}}\left|f\left(r_{1} \zeta_{1}, r_{2} \zeta_{2}\right)\right|^{p} d s\left(\zeta_{1}\right) d s\left(\zeta_{2}\right) \\
& =\int_{s_{2}}\left(\int_{s_{1}}\left|f\left(r_{1} \zeta_{1}, r_{2} \zeta_{2}\right)\right|^{p} d s\left(\zeta_{1}\right)\right) d s\left(\zeta_{2}\right)
\end{aligned}
$$

where $0 \leq r_{1}, r_{2}<1$. For fixed $r_{2}$ and $\zeta_{2}$, the inner integral

$$
\int_{s_{1}}\left|f\left(r_{1} \zeta_{1}, r_{2} \zeta_{2}\right)\right|^{p} d s\left(\zeta_{1}\right)
$$

in (5) is $f_{z_{2}}\left(z_{1}\right)=f\left(z_{1}, z_{2}\right)$, which corresponding to a unitary holomorphic function $M_{p}^{p}\left(f_{r_{2} \zeta_{2}}, r_{1}\right)$. Hence $M_{p}^{p}\left(f, r_{1}, r_{2}\right)$ is increasing with respect to $r_{1}$, i. e.

$$
\frac{\partial M_{p}^{p}\left(f, r_{1}, r_{2}\right)}{\partial r_{1}} \geq 0 .
$$

Similarly, $M_{p}^{p}\left(f, r_{1}, r_{2}\right)$ is an increasing function of $r_{2}$. In particular, since $f_{z_{2}}\left(z_{1}\right)=f\left(z_{1}, z_{2}\right)$ is a non-constant 
function, $M_{p}^{p}\left(f, r_{1}, r_{2}\right)$ is a strictly increasing function of $r_{2}$, so

$$
\frac{\partial M_{p}^{p}\left(f, r_{1}, r_{2}\right)}{\partial r_{2}}>0
$$

Now from $M_{p}^{p}(f, r)=M_{p}^{p}\left(f, r_{1}, r_{2}\right)$ and (6),(7) we get

$$
\frac{d M_{p}^{p}(f, r)}{d r}=\left.\left(\frac{\partial M_{p}^{p}\left(f, r_{1}, r_{2}\right)}{\partial r_{1}}+\frac{\partial M_{p}^{p}\left(f, r_{1}, r_{2}\right)}{\partial r_{2}}\right)\right|_{r_{1}=r_{2}=r}>0
$$

which implies that $M_{p}(f, r)$ is strictly increasing.

We continue to prove the following conclusion of $M_{p, \alpha}(f, r)$.

Theorem 2.2 For $0<p<\infty$ and $\alpha$ being a real number.

Let $f(z)$ be a holomorphic function on $\mathrm{P}_{2}$. Then the function $r \mapsto M_{p, \alpha, \beta}(f, r)$ is an increasing function on the interval $[0,1)$. In particular, if $f$ is non-constant, then $f$ is strictly increasing.

Proof: We may rewrite the integral as follows

$$
\begin{aligned}
& \int_{r \mathrm{P}_{2}}|f(z)|^{p} d v_{\alpha}(z) \\
& =\int_{0}^{r} \int_{0}^{r}\left(1-\left|\rho_{1}\right|^{2}\right)^{\alpha}\left(1-\left|\rho_{2}\right|^{2}\right)^{\alpha} \rho_{1} \rho_{2} \int_{s_{1} \times S_{2}}\left|f\left(\rho_{1} e^{i \theta}, \rho_{2} e^{i \phi}\right)\right|^{p} \frac{d \theta d \phi}{\pi^{2}} d \rho_{1} d \rho_{2}
\end{aligned}
$$

Then

$$
\begin{aligned}
& \frac{d}{d r} \mathrm{I}(v)=r\left(1-r^{2}\right) \int_{0}^{r}\left(1-\left|\rho_{1}\right|^{2}\right)^{\alpha} \rho_{1} \int_{\mathrm{T}^{2}}\left|f\left(\rho_{1} \zeta, r \zeta\right)\right|^{p} d \sigma(\zeta) d \rho_{1} \\
& +r\left(1-r^{2}\right) \int_{0}^{r}\left(1-\left|\rho_{2}\right|^{2}\right)^{\alpha} \rho_{2} \int_{\mathrm{T}^{2}}\left|f\left(r \zeta, \rho_{2} \zeta\right)\right|^{p} d \sigma(\zeta) d \rho_{2}
\end{aligned}
$$

where $\frac{d \theta d \phi}{\pi^{2}}=d \sigma\left(\zeta^{\prime}\right)$ is on torus and $\zeta=\left(\zeta_{1}, \zeta_{2}\right) \in \mathrm{T}^{2}$.

Similarly, since

$$
v_{\alpha}\left(r \mathrm{P}_{2}\right)=\int_{r \mathrm{P}_{2}} d v_{\alpha}(z)=4 \int_{0}^{r} \int_{0}^{r}\left(1-\rho_{1}\right)^{\alpha} \rho_{1}\left(1-\rho_{2}{ }^{2}\right)^{\alpha} \rho_{2} d \rho_{1} d \rho_{2}
$$

we have

$$
\frac{d}{d r} v_{\alpha}\left(r \mathrm{P}_{2}\right)=\frac{4 r}{\alpha+1}\left(1-r^{2}\right)^{\alpha}\left[-\left(1-r^{2}\right)^{\alpha+1}+1\right]
$$

Then the derivative of the function $M_{p, \alpha}^{p}(f, r)$ with respect to $r$ is given by

$$
\begin{aligned}
& 4 \int_{0}^{r} \int_{0}^{r}\left(1-\rho_{1}^{2}\right)^{\alpha} \rho_{1}\left(1-\rho_{2}^{2}\right)^{\alpha} \rho_{2}\left[\begin{array}{l}
r\left(1-r^{2}\right)^{\alpha} \int_{0}^{r}\left(1-\rho_{1}^{2}\right)^{\alpha} \rho_{1} \int_{\mathrm{T}^{2}}\left|f\left(\rho_{1} \zeta_{1}, r \zeta_{2}\right)\right|^{p} d \sigma(\zeta) d \rho_{1} \\
+r\left(1-r^{2}\right)^{\alpha} \int_{0}^{r}\left(1-\rho_{2}^{2}\right)^{\alpha} \rho_{2} \int_{\mathrm{T}^{2}}\left|f\left(r \zeta_{1}, \rho_{2} \zeta_{2}\right)\right|^{p} d \sigma(\zeta) d \rho_{2}
\end{array}\right] \\
& -\int_{0}^{r} \int_{0}^{r}\left(1-\left|\rho_{1}\right|^{2}\right)^{\alpha}\left(1-\left|\rho_{2}\right|^{2}\right)^{\alpha} \rho_{1} \rho_{2} \int_{\mathrm{T}^{2}}\left|f\left(\rho_{1} \zeta_{1}, \rho_{2} \zeta_{2}\right)\right|^{p} d \sigma(\zeta) d \rho_{1} d \rho_{2} \\
& {\left[4 \int_{0}^{r}\left(1-\rho_{1}^{2}\right)^{\alpha} \rho_{1} d \rho_{1} r\left(1-r^{2}\right)^{\alpha}+4 \int_{0}^{r}\left(1-\rho_{2}{ }^{2}\right)^{\alpha} \rho_{2} d \rho_{2} r\left(1-r^{2}\right)^{\alpha}\right]}
\end{aligned}
$$

where

$$
\frac{d}{d r}\left(\frac{1}{v_{\alpha}\left(r \mathrm{P}_{2}\right)} \int_{r \mathrm{P}_{2}}|f(z)|^{p} d v_{\alpha}(z)\right) \sim v_{\alpha}\left(r \mathrm{P}_{2}\right) \frac{d \mathrm{I}(v)}{d r}-\mathrm{I}(v) \frac{d v_{\alpha}\left(r \mathrm{P}_{2}\right)}{d r} .
$$

function with respect to $r$, and if $f$ is not a constant function, it is strictly increasing.

\section{Logarithmical Convexity}

Since

$$
\begin{aligned}
& \int_{\mathrm{T}^{2}}\left|f\left(\rho_{1} \zeta_{1}, r \zeta_{2}\right)\right|^{p} d \sigma(\zeta)>\int_{\mathrm{T}^{2}}\left|f\left(\rho_{1} \zeta_{1}, \rho_{2} \zeta_{2}\right)\right|^{p} d \sigma(\zeta) \\
& \int_{\mathrm{T}^{2}}\left|f\left(r \zeta_{1}, \rho_{2} \zeta_{2}\right)\right|^{p} d \sigma(\zeta)>\int_{\mathrm{T}^{2}}\left|f\left(\rho_{1} \zeta_{1}, \rho_{2} \zeta_{2}\right)\right|^{p} d \sigma(\zeta)
\end{aligned}
$$

We now consider the $\operatorname{logarithmic}$ function $\log M_{p}(f, r)$ on the Hardy space.

Theorem 3.1 Let $0<p<\infty$ and $f$ be a holomorphic function in the Hardy space $\mathrm{H}_{p}\left(\mathrm{P}_{2}\right)$. Then the function $\log M_{p}(f, r)$ is a convex function with respect to $\log r$ with $r \in(0,1)$.

Proof: It is similar to the proof of Theorem 1.6 in Duren's book [4], w suppose for any real number $\lambda$ that

$$
\begin{aligned}
m_{p}\left(f, \lambda, r_{1}, r_{2}\right) & =\frac{r_{1}^{\lambda}}{2 \pi} \frac{r_{2}^{\lambda}}{2 \pi} \int_{0}^{2 \pi} \int_{0}^{2 \pi}\left|f_{r_{2} \zeta_{2}}\left(r_{1} \zeta_{1}, r_{2} \zeta_{2}\right)\right|^{p} d \theta_{1} d \theta_{2} \\
& =\frac{1}{2 \pi} \frac{1}{2 \pi} \int_{0}^{2 \pi} \int_{0}^{2 \pi}\left|r_{1} \zeta_{1}\right|^{\lambda}\left|r_{2} \zeta_{2}\right|^{\lambda}\left|f_{z}\left(r_{1} \zeta_{1}, r_{2} \zeta_{2}\right)\right|^{p} d \theta_{1} d \theta_{2} .
\end{aligned}
$$

and the equality in (14) holds only if $f$ is a constant function.

Therefore, $M_{p, \alpha}^{p}(f, r)$ or $M_{p, \alpha}(f, r)$ is an increasing

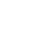

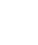


Then

$$
M_{p}\left(f, r_{1}, r_{2}\right)=m_{p}\left(f, 0, r_{1}, r_{2}\right)
$$
$\left|z_{1}\right|^{\lambda}\left|z_{2}\right|^{\lambda}\left|f_{z_{2}}\left(z_{1}, z_{2}\right)\right|^{p} \quad$ is a pluriharmonic function on $\left\{\left(z_{1}, z_{2}\right) \in \mathrm{P}_{2}, r^{\prime}<\left|z_{1}\right|,\left|z_{2}\right|<r^{\prime \prime}\right\}$, so it exists a pluriharmonic function $U\left(z_{1}, z_{2}\right)$ on $\left\{\left(z_{1}, z_{2}\right) \in \mathrm{P}_{2}, r^{\prime}<\left|z_{1}\right|,\left|z_{2}\right|<r^{\prime \prime}\right\}$ and equal to $\left|z_{1}\right|^{\lambda}\left|z_{2}\right|^{\lambda}\left|f_{z_{2}}\left(z_{1}, z_{2}\right)\right|^{p}$ on the boundary. By the relationship between the subharmonic function and the harmonic function, we have

$$
m_{p}\left(f, \lambda, r_{1}, r_{2}\right) \leq \frac{1}{2 \pi} \frac{1}{2 \pi} \int_{0}^{2 \pi} \int_{0}^{2 \pi} U\left(r_{1} e^{i \theta_{1}}, r_{2} e^{i \theta_{2}}\right) d \theta_{1} d \theta_{2}, r^{\prime}<r_{1}, r_{2}<r^{\prime \prime}
$$

The second-order mixed partial derivative of the right-hand side of (16) is obtained and then the order of integral and partial derivative is exchanged to get

$$
\frac{\partial}{\partial r_{2}} \frac{\partial}{\partial r_{1}}\left(\int_{0}^{2 \pi} \int_{0}^{2 \pi} U\left(r_{1} e^{i \theta_{1}}, r_{2} e^{i \theta_{2}}\right) d \theta_{1} d \theta_{2}\right)=\frac{\partial}{\partial r_{2}} \int_{0}^{2 \pi} \frac{1}{r_{1}} \int_{0}^{2 \pi} \frac{\partial}{\partial n_{1}} U\left(\zeta_{1}, r_{2} e^{i \theta_{2}}\right) d s_{1} d \theta_{2}
$$

In the upper formula, $\partial / \partial n_{1}$ represents the normal partial derivative of the first variable, $\mathrm{U}_{1} \cos \theta_{1} d \theta_{1}+\mathrm{U}_{2} \sin \theta_{1} d \theta_{1}=\frac{1}{r_{1}} \frac{\partial \mathrm{U}}{\partial n_{1}} d\left(\theta_{1} r_{1}\right)$ and $d s$. By green's formula and since $U$ is harmonic, we have that the inner interal of (17) ia an univariate function of $r_{2} e^{i \theta_{2}}$. Let

$$
\int_{|\zeta|=r_{1}} \frac{\partial U}{\partial n_{1}} d s=g\left(r_{2} e^{i \theta_{2}}\right)
$$

then

$$
\frac{1}{2 \pi} \frac{1}{2 \pi} \int_{0}^{2 \pi} \int_{0}^{2 \pi} U\left(r e^{i \theta_{1}}, r e^{i \theta_{2}}\right) d \theta_{1} d \theta_{2}=\mathrm{A}(\log r)^{2}+\left(\mathrm{B}_{1}+\mathrm{B}_{2}\right) \log r+c=\mathrm{A}(\log r)^{2}+\mathrm{B} \log r+c, r^{\prime}<r<r^{\prime \prime} .
$$

The above formula is obviously a convex function of $\log r$, so (16) yields that $m_{p}(f, \lambda, r, r)$ is logarithmically convex, that is,

$$
\log m_{p}\left(f, \lambda, \frac{r^{\prime}+r^{\prime \prime}}{2}, \frac{r^{\prime}+r^{\prime \prime}}{2}\right)<\frac{1}{2}\left[\log m_{p}\left(f, \lambda, r^{\prime}, r^{\prime}\right)+\log m_{p}\left(f, \lambda, r^{\prime \prime}, r^{\prime \prime}\right)\right]
$$

Similar to the discussion in Xiao-Zhu [2], (21) and Duren [4, p. 10] implies that $\log m_{p}(f, r)$ is also a convex function of $\log r$, which completes the proof.

Remark. Inspired by the logaithmical convexity in Theorem 3.1 and the weightless integral mean $M_{p}(f, r)$, Xiao and Zhu naturally consider the logarithmic convexity of the volume integral mean $M_{p, \alpha}(f, r)$ of $\log r$ on a unit sphere (see [2]). They found that the logarithmical convexity problem is more complicated at this time, in fact, for some $\alpha$, $M_{p, \alpha}(f, r)$ is logarithmically convex, while for others it is logarithmically concave. On multiple cylinders, the problem becomes much more complicated because of the correlation between the two parameters $\alpha$ and $\beta$. We now give some examples below.

Example 3.2. Let $\mathrm{f}$ be a holomorphic function on $\mathrm{P}_{2}$. Then for $1 \leq p<\infty, \log M_{p, 0}(f, r)$ is a convex function of $\log r$.
Proof: Notice first that we have

$$
M_{p, 0}(f, r)=\left[\int_{\mathrm{P}_{2}}|f(r \zeta)|^{p} d v(\zeta)\right]^{1 / p}
$$

and we may rewrite it as

$$
M_{p, 0}(f, r)=\int_{0}^{1} \rho_{1} d \rho_{1} \int_{0}^{1} \rho_{2} d \rho_{2} \int_{\partial \mathrm{P}_{2}}\left|f\left(r \rho_{1} \zeta_{1}, r \rho_{2} \zeta_{2}\right)\right|^{p} d \sigma(\zeta)
$$

By Theorem 3.1, the logarithm of the innermost integral of (22) is logarithmically convex, so by the method in [6] we obtain that $\log M_{p, 0}(f, r)$ is a convex function of $\log r$.

Example 3.3. For $p=2, \alpha=1$. Let $f(z)=z_{1} z_{2}$. Then $\log M_{p, \alpha}(f, r)$ is a concave function of $\log r$.

Proof: By [2, Example 10], the logarithms of volume mean integrals $M_{p, \alpha}\left(z_{1}, r\right)$ and $M_{p, \alpha}\left(z_{2}, r\right)$ on a unit disk are concave function of $\log r$. Then simple calculations give 


$$
\log M_{p, \alpha}(f, r)=\log M_{p, \alpha}\left(z_{1}, r\right)+\log M_{p, \alpha}\left(z_{2}, r\right) .
$$

Hence $\log M_{p, \alpha}(f, r)$ is concave function of $\log r$.

\section{Conclusion}

The proof of the main theorem (Theorem 3.1) is different from that of Xiao-Zhu $[2,7-15]$. And we only work on the case of $\alpha=\beta$. The problem becomes much more complicated for the case of different because parameters $\alpha$ and $\beta$. In addition to the lack of symmetry on multiple cylinders mentioned in the proof which makes the tool of slice function unusable, it can also be seen from (20) that the proof here does not deal with the case on three-dimensional multiple cylinders.

\section{Funding}

This study was funded by the National Natural Science Foundation of China through Grant Nos. 11802208 and the Tianjin Education Scientific Research through Grant Nos. 2019KJ140.

\section{Acknowledgements}

The authors would like to thank the referee for his valuable suggestions, which helped us to improve the presentation of the paper. The authors gratefully acknowledge the support of the National Natural Science Foundation of China (NNSFC) through Grant Nos. 11802208 and the Tianjin Education Scientific Research through Grant Nos. 2019KJ140.

\section{References}

[1] Zhu K. H. Space of Holomorphic Functions in the Unit ball [M]. New York: Springer-verlag, 2005.
[2] Xiao J, Zhu K. H. Volume integral means of holomorphic functions [J]. Proc. American Mathematical Society, 2011, 139 (4): 1455-1465.

[3] Liu Hua. On the linear extreme of bergman space on polydisc [J]. Of Xuzhou Normal Uni: Natural Sciences, 2003, 21 (2): 1-4. (Chinese).

[4] Duren P. Theory of Hp Space [M]. New York: Academic Press, 1970.

[5] Shi Jihuai. Foundations of function theory of several complex variables [M]. Bei Jing: Higher Education Press, 1996. (Chinese).

[6] Taylor A E. New proofs of some theorems of Hardy by Banach space methods [J], Math. Magazine, 23 (1950), 115-124.

[7] Zhu K. H. (2004). Translating Inequalities between Hardy and Bergman Spaces [J]. American Mathematical Monthly. 111. $10.2307 / 4145071$.

[8] Zhu K. H. (1990). On certain unitary operators and composition operators [J]. 10.1090/pspum/051.2/1077459.

[9] Zhu K. H. (2005). Spaces of Holomorphic Functions in the Unit Ball [J]. Grad Texts in Math. 10.1007/0-387-27539-8.

[10] Zhu K. H. (2015). Singular Integral Operators on the Fock Space. Integral Equations and Operator Theory. 81. $10.1007 / \mathrm{s} 00020-015-2222-9$.

[11] Xiao Jie. (2019). Prescribing Capacitary Curvature Measures on Planar Convex Domains [J]. The Journal of Geometric Analysis. 10.1007/s12220-019-00180-9.

[12] Xiao Jie. (2015). On the variational \$p\$-capacity problem in the plane [J]. Communications on Pure and Applied Analysis. 14. 959-968. 10.3934/cpaa.2015.14.959.

[13] Xiao Jie. (2017). The p-Affine Capacity Redux [J]. The Journal of Geometric Analysis. 27. 10.1007/s12220-017-9785-4.

[14] Xiao Jie. (2015). The \$\$p\$ $\$$-Affine Capacity [J]. The Journal of Geometric Analysis. 26. 10.1007/s12220-015-9579-5.

[15] Xiao, Jie. (2014). Optimal geometric estimates for fractional Sobolev capacities [J]. Comptes Rendus Mathematique. 354. 10.1016/j.crma.2015.10.014. 